International Journal of Pure and Applied Mathematics

Volume 110 No. $3 \quad$ 2016, 477-487

ISSN: 1311-8080 (printed version); ISSN: 1314-3395 (on-line version)

url: http://www.ijpam.eu

doi: $10.12732 / \mathrm{ijpam} . v 110 \mathrm{i} 3.8$

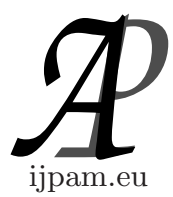

\title{
SOME FIXED POINT THEOREMS FOR COMPATIBLE MAPPINGS OF TYPES IN MULTIPLICATIVE METRIC SPACES
}

Chahn Yong Jung ${ }^{1}$, Parveen Kumar ${ }^{2}$, Sanjay Kumar ${ }^{2}$, Shin Min Kang 3 §

${ }^{1}$ Department of Business Administration

Gyeongsang National University

Jinju, 52828, KOREA

${ }^{2}$ Departement of Mathematics

Deenbandhu Chhotu Ram University of Science and Technology

Murthal, Sonepat, 131039, Haryana, INDIA

${ }^{3}$ Department of Mathematics and RINS

Gyeongsang National University

Jinju, 52828, KOREA

Abstract: In this paper, we proved the common fixed point theorems for compatible mappings of types in multiplicative metric spaces.

AMS Subject Classification: $47 \mathrm{H} 10,54 \mathrm{H} 25$

Key Words: multiplicative metric spaces, compatible mappings of type $(R)$, of type $(K)$, of type $(E)$, reciprocally continuous

\section{Introduction and Preliminaries}

It is well know that the set of positive real numbers $\mathbb{R}_{+}$is not complete according to the usual metric. To overcome this problem, in 2008, Bashirov et al. [1] introduced the concept of multiplicative metric spaces as follows:

Received: $\quad$ August 11, 2016

Revised: $\quad$ September 11, 2016

Published: $\quad$ November 5, 2016

$\S$ Correspondence author (c) 2016 Academic Publications, Ltd. url: www.acadpubl.eu 
Definition 1.1. Let $X$ be a nonempty set. A multiplicative metric is a mapping $d: X \times X \rightarrow \mathbb{R}_{+}$satisfying the following conditions:

(i) $d(x, y) \geq 1$ for all $x, y \in X$ and $d(x, y)=1$ if and only if $x=y$;

(ii) $d(x, y)=d(y, x)$ for all $x, y \in X$;

(iii) $d(x, y) \leq d(x, z) \cdot d(z, y)$ for all $x, y, z \in X$ (multiplicative triangle inequality).

Then the mapping $d$ together with $X$, that is, $(X, d)$ is a multiplicative metric space.

Example 1.2. ([6]) Let $\mathbb{R}_{+}^{n}$ be the collection of all $n$-tuples of positive real numbers. Let $d^{*}: \mathbb{R}_{+}^{n} \times \mathbb{R}_{+}^{n} \rightarrow \mathbb{R}$ be defined as follows:

$$
d^{*}(x, y)=\left|\frac{x_{1}}{y_{1}}\right|^{*} \cdot\left|\frac{x_{2}}{y_{2}}\right|^{*} \cdots\left|\frac{x_{n}}{y_{n}}\right|^{*},
$$

where $x=\left(x_{1}, \ldots, x_{n}\right), y=\left(y_{1}, \ldots, y_{n}\right) \in \mathbb{R}_{+}^{n}$ and $|\cdot|^{*}: \mathbb{R}_{+} \rightarrow \mathbb{R}_{+}$is defined by

$$
|a|^{*}= \begin{cases}a & \text { if } a \geq 1 \\ \frac{1}{a} & \text { if } a<1 .\end{cases}
$$

Then it is obvious that all conditions of a multiplicative metric are satisfied. Therefore $\left(\mathbb{R}_{+}^{n}, d^{*}\right)$ is a multiplicative metric space.

One can refer to [6] for detailed a multiplicative metric topology.

Definition 1.3. Let $(X, d)$ be a multiplicative metric space. Then a sequence $\left\{x_{n}\right\}$ in $X$ is said to be

(1) a multiplicative convergent to $x$ if for every multiplicative open ball $B_{\epsilon}(x)=\{y \mid d(x, y)<\epsilon\}, \epsilon>1$, there exists $N \in \mathbb{N}$ such that $x_{n} \in B_{\epsilon}(x)$ for all $n \geq N$, that is, $d\left(x_{n}, x\right) \rightarrow 1$ as $n \rightarrow \infty$.

(2) a multiplicative Cauchy sequence if for all $\epsilon>1$, there exists $N \in \mathbb{N}$ such that $d\left(x_{n}, x_{m}\right)<\epsilon$ for all $m, n \geq N$, that is, $d\left(x_{n}, x_{m}\right) \rightarrow 1$ as $n, m \rightarrow \infty$.

(3) We call a multiplicative metric space complete if every multiplicative Cauchy sequence in it is multiplicative convergent to $x \in X$.

In 2012, Özavsar and Çevikel [6] gave the concept of multiplicative contractive mappings and proved some fixed point theorem of such mappings in a multiplicative metric space.

Definition 1.4. Let $f$ be a mapping of a multiplicative metric space $(X, d)$ into itself. Then $f$ is said to be a multiplicative contraction if there exists a real number $\lambda \in[0,1)$ such that

$$
d(f x, f y) \leq d^{\lambda}(x, y) \quad \text { for all } x, y \in X .
$$


In 2015, Kang et al. [2, 3] introduced the notion of compatible mappings and its variants in a multiplicative metric space as follows:

Definition 1.5. Let $f$ and $g$ be two mappings of a multiplicative metric space $(X, d)$ into itself. Then $f$ and $g$ are called

(1) compatible if

$$
\lim _{n \rightarrow \infty} d\left(f g x_{n}, g f x_{n}\right)=1,
$$

whenever $\left\{x_{n}\right\}$ is a sequence in $X$ such that $\lim _{n \rightarrow \infty} f x_{n}=\lim _{n \rightarrow \infty} g x_{n}=t$ for some $t \in X$.

(2) compatible of type $(P)$ if

$$
\lim _{n \rightarrow \infty} d\left(f f x_{n}, g g x_{n}\right)=1,
$$

whenever $\left\{x_{n}\right\}$ is a sequence in $X$ such that $\lim _{n \rightarrow \infty} f x_{n}=\lim _{n \rightarrow \infty} g x_{n}=t$ for some $t \in X$.

(3) compatible of type $(R)$ if

$$
\lim _{n \rightarrow \infty} d\left(f g x_{n}, g f x_{n}\right)=1 \text { and } \lim _{n \rightarrow \infty} d\left(f f x_{n}, g g x_{n}\right)=1,
$$

whenever $\left\{x_{n}\right\}$ is a sequence in $X$ such that $\lim _{n \rightarrow \infty} f x_{n}=\lim _{n \rightarrow \infty} g x_{n}=t$ for some $t \in X$,

(4) compatible of type $(K)$ if

$$
\lim _{n \rightarrow \infty} d\left(f f x_{n}, g t\right)=1 \text { and } \lim _{n \rightarrow \infty} d\left(g g x_{n}, f t\right)=1,
$$

whenever $\left\{x_{n}\right\}$ is a sequence in $X$ such that $\lim _{n \rightarrow \infty} f x_{n}=\lim _{n \rightarrow \infty} g x_{n}=t$ for some $t \in X$,

(5) compatible of type $(E)$ if

$$
\lim _{n \rightarrow \infty} f f x_{n}=\lim _{n \rightarrow \infty} f g x_{n}=g t \quad \text { and } \quad \lim _{n \rightarrow \infty} g g x_{n}=\lim _{n \rightarrow \infty} g f x_{n}=f t,
$$

whenever $\left\{x_{n}\right\}$ is a sequence in $X$ such that $\lim _{n \rightarrow \infty} f x_{n}=\lim _{n \rightarrow \infty} g x_{n}=t$ for some $t \in X$.

Remark 1.6. Obviously, compatible mappings of type $(R)$ are also compatible mappings and compatible mappings of type $(P)$.

Definition 1.7. ([3]) Let $f$ and $g$ be mappings of a multiplicative metric space $(X, d)$. Then $f$ and $g$ are called reciprocally continuous if

$$
\lim _{n \rightarrow \infty} f g x_{n}=f t \text { and } \lim _{n \rightarrow \infty} g f x_{n}=f t,
$$

whenever $\left\{x_{n}\right\}$ is a sequence in $X$ such that $\lim _{n \rightarrow \infty} f x_{n}=\lim _{n \rightarrow \infty} g x_{n}=t$ for some $t \in X$, 
Now we give some properties related to compatible mappings of types in a multiplicative metric space, see [3].

Proposition 1.8. Let $f$ and $g$ compatible mappings of type $(E)$ of a multiplicative metric space $(X, d)$ itself. Let one of $f$ and $g$ be continuous. Suppose that $\lim _{n \rightarrow \infty} f x_{n}=\lim _{n \rightarrow \infty} g x_{n}=t$ for some $t \in X$. Then

(a) $f t=g t$ and $\lim _{n \rightarrow \infty} f f x_{n}=\lim _{n \rightarrow \infty} g g x_{n}=\lim _{n \rightarrow \infty} f g x_{n}=\lim _{n \rightarrow \infty} g f x_{n}$.

(b) If there exists $u \in X$ such that $f u=g u=t$, then $f g u=g f u$.

\section{Main Results}

Now we give the following theorem for compatible mappings of type $(R)$.

Theorem 2.1. Let $A, B, S$ and $T$ be mappings of a complete multiplicative metric space $(X, d)$ satisfying the following conditions

$$
S X \subset B X, \quad T X \subset A X
$$

$$
d(S x, T y) \leq M^{\lambda}(x, y)
$$

for each $x, y \in X$ and $\lambda \in(0,1 / 2)$, where

$$
\begin{aligned}
& M(x, y) \\
& =\max \{d(A x, S x), d(B y, T y), d(B y, A x), \\
& (d(A x, T y) \cdot d(B y, S x))^{1 / 2}, \min \left\{\frac{d(A x, S x) \cdot d(B y, T y)}{d(A x, B y)},\right. \\
& \left.\left.\quad \frac{d(A x, T y) \cdot d(B y, S x)}{d(A x, B y)}, \frac{d(A x, T y) \cdot d(B y, S x)}{d(S x, T y)}\right\}\right\} ;
\end{aligned}
$$

$\left(C_{3}\right)$ one of $A, B, S$ and $T$ is continuous.

Assume that the pairs $A, S$ and $B, T$ are compatible of type $(R)$. Then $A, B, S$ and $T$ have a unique common fixed point.

Proof. It follows from Remark 1.6 and [4, Theorem 2.1] or [5, Theorem 2.4].

Next we give the following theorem for compatible mappings of type $(K)$. 
Theorem 2.2. Let $A, B, S$ and $T$ be mappings of a complete multiplicative metric space $(X, d)$ into itself satisfying the conditions $\left(C_{1}\right)$ and $\left(C_{2}\right)$.

Assume that the pairs $A, S$ and $B, T$ are compatible of type $(K)$, the pairs $A, S$ and $B, T$ are reciprocally continuous. Then $A, B, S$ and $T$ have a unique common fixed point in $X$.

Proof. Let $x_{0} \in X$ be an arbitrary point. Since $S X \subset B X$ and $T X \subset A X$, there exists $x_{1} \in X$ such that $S x_{0}=B x_{1}=y_{0}$ and for this point $x_{1}$, there exists $x_{2} \in X$ such that $T x_{1}=A x_{2}=y_{1}$. Continuing in this way, we can construct a sequence $\left\{y_{n}\right\}$ such that

$$
y_{2 n+1}=T x_{2 n+1}=A x_{2 n+2} ; \quad y_{2 n}=S x_{2 n}=B x_{2 n+1} .
$$

From the proof of $\left[4\right.$, Theorem 2.2], $\left\{y_{n}\right\}$ is a multiplicative Cauchy sequence in $X$ and hence it converges to some point $z \in X$. Consequently, the subsequence $\left\{S x_{2 n}\right\},\left\{B x_{2 n+1}\right\},\left\{T x_{2 n+1}\right\}$ and $\left\{A x_{2 n}\right\}$ of sequence $\left\{y_{n}\right\}$ also converges to $z$.

Since the pairs $A, S$ and $B, T$ are compatible of type $(K)$ on $X$, we have

$$
A A x_{2 n} \rightarrow S z, \quad S S x_{2 n} \rightarrow A z, \quad B B x_{2 n+1} \rightarrow T z, \quad T T x_{2 n+1} \rightarrow B z
$$

as $n \rightarrow \infty$.

Now putting $x=S x_{2 n}$ and $y=T x_{2 n+1}$ in $\left(C_{2}\right)$, we have

$$
d\left(S S x_{2 n}, T T x_{2 n+1}\right) \leq M^{\lambda}\left(S x_{2 n}, T x_{2 n+1}\right)
$$

where

$$
\begin{aligned}
M\left(S x_{2 n}, T x_{2 n+1}\right) & \max \left\{d\left(A S x_{2 n}, S S x_{2 n}\right), d\left(B T x_{2 n+1}, T T x_{2 n+1}\right), d\left(B T x_{2 n+1}, A S x_{2 n}\right),\right. \\
& \left(d\left(A S x_{2 n}, T T x_{2 n+1}\right) \cdot d\left(B T x_{2 n+1}, S S x_{2 n}\right)\right)^{1 / 2} \\
& \min \left\{\frac{d\left(A S x_{2 n}, S S x_{2 n}\right) \cdot d\left(B T x_{2 n+1}, T T x_{2 n+1}\right)}{d\left(A S x_{2 n}, B T x_{2 n+1}\right)},\right. \\
& \frac{d\left(A S x_{2 n}, T T x_{2 n+1}\right) \cdot d\left(B T x_{2 n+1}, S S x_{2 n}\right)}{d\left(A S x_{2 n}, B T x_{2 n+1}\right)}, \\
& \left.\left.\frac{d\left(A S x_{2 n}, T T x_{2 n+1}\right) \cdot d\left(B T x_{2 n+1}, S S x_{2 n}\right)}{d\left(S S x_{2 n}, T T x_{2 n+1}\right)}\right\}\right\}
\end{aligned}
$$

Letting $n \rightarrow \infty$ and using reciprocal continuity of the pairs $A, S$ and $B, T$, we 
have

$$
\begin{aligned}
& \lim _{n \rightarrow \infty} M\left(S x_{2 n}, x_{2 n+1}\right) \\
& =\max \{d(A z, A z), d(B z, B z), d(B z, A z), d(B z, A z), \\
& \left.\quad \min \left\{\frac{1}{d(B z, A z)}, d(A z, B z), d(A z, B z)\right\}\right\} \\
& =d(B z, A z) .
\end{aligned}
$$

Hence we have

$$
d(A z, B z) \leq d^{\lambda}(B z, A z),
$$

which implies that $A z=B z$.

Next putting $x=z$ and $y=T x_{2 n+1}$ in $\left(C_{2}\right)$, we have

$$
d\left(S z, T T x_{2 n+1}\right) \leq M^{\lambda}\left(z, T x_{2 n+1}\right)
$$

where

$$
\begin{aligned}
M\left(z, T x_{2 n+1}\right) & \max \left\{d(B z, S z), d\left(B T x_{2 n+1}, T T x_{2 n+1}\right), d\left(B T x_{2 n+1}, A z\right),\right. \\
( & \left.d\left(A z, T T x_{2 n+1}\right) \cdot d\left(B T x_{2 n+1}, S z\right)\right)^{1 / 2}, \\
& \min \left\{\frac{d(A z, S z) \cdot d\left(B T x_{2 n+1}, T T x_{2 n+1}\right)}{d\left(A z, B T x_{2 n+1}\right)},\right. \\
& \frac{d\left(A z, T T x_{2 n+1}\right) \cdot d\left(B T x_{2 n+1}, S z\right)}{d\left(A z, B T x_{2 n+1}\right)}, \\
& \left.\left.\frac{d\left(A z, T T x_{2 n+1}\right) \cdot d\left(B T x_{2 n+1}, S z\right)}{d\left(S z, T T x_{2 n+1}\right)}\right\}\right\} .
\end{aligned}
$$

Letting $n \rightarrow \infty$ and using reciprocal continuity of the pairs $A, S$ and $B, T$, we have

$$
\begin{aligned}
& \lim _{n \rightarrow \infty} M\left(z, T x_{2 n+1}\right) \\
& =\max \{d(B z, S z), d(B z, B z), d(B z, B z), d(B z, S z), \\
& \quad \min \{d(B z, S z), d(B z, S z), d(B z, S z)\}\} \\
& =d(B z, S z) .
\end{aligned}
$$

This implies that $S z=B z$.

Next putting $x=z$ and $y=z$ in inequality $\left(C_{2}\right)$, we have

$$
d(S z, T z) \leq M^{\lambda}(z, z),
$$


where

$$
\begin{aligned}
& M(z, z) \\
& =\max \{d(A z, S z), d(B z, T z), d(B z, A z) \\
& \quad(d(A z, T z) \cdot d(B z, S z))^{1 / 2}, \min \left\{\frac{d(A z, S z) \cdot d(B z, T z)}{d(A z, B z)},\right. \\
& \left.\left.\quad \frac{d(A z, T z) \cdot d(B z, S z)}{d(A z, B z)}, \frac{d(A z, T z) \cdot d(B z, S z)}{d(S z, T z)}\right\}\right\} \\
& =d(T z, S z)
\end{aligned}
$$

which implies that $S z=T z$. Hence we have $A z=B z=S z=T z$.

Next, putting $x=x_{2 n}$ and $y=z$ in inequality $\left(C_{2}\right)$, we have

$$
d\left(S x_{2 n}, T z\right) \leq M^{\lambda}\left(x_{2 n}, z\right)
$$

where

$$
\begin{aligned}
& M\left(x_{2 n}, z\right) \\
& =\max \left\{d\left(A x_{2 n}, S x_{2 n}\right), d(B z, T z), d\left(B z, A x_{2 n}\right)\right. \\
& \quad\left(d\left(A x_{2 n}, T z\right) \cdot d\left(B z, S x_{2 n}\right)\right)^{1 / 2}, \min \left\{\frac{d\left(A x_{2 n}, S x_{2 n}\right) \cdot d(B z, T z)}{d\left(A x_{2 n}, B z\right)},\right. \\
& \left.\left.\quad \frac{d\left(A x_{2 n}, T z\right) \cdot d\left(B z, S x_{2 n}\right)}{d\left(A x_{2 n}, B z\right)}, \frac{d\left(A x_{2 n}, T z\right) \cdot d\left(B z, S x_{2 n}\right)}{d\left(S x_{2 n}, T z\right)}\right\}\right\} .
\end{aligned}
$$

Letting $n \rightarrow \infty$, we get

$$
\begin{aligned}
& \lim _{n \rightarrow \infty} M\left(x_{2 n}, z\right) \\
& =\max \{d(z, z), d(T z, T z), d(T z, z), d(T z, z), \\
& \left.\quad \min \left\{\frac{1}{d(z, T z)}, d(z, T z), 1\right\}\right\} \\
& =d(T z, z) .
\end{aligned}
$$

This implies that $T z=z$ and hence $z=A z=B z=S z=T z$. Hence $z$ is a common fixed point of $A, S, B$ and $T$.

Finally, suppose that $z$ and $w$ are two common fixed points of $A, B, S$ and $T$. 
Now putting $x=z$ and $y=w$ in $\left(C_{2}\right)$

$$
d(z, w)=d^{\lambda}(S z, T w) \leq M^{\lambda}(z, w)
$$

where

$$
\begin{aligned}
& M(z, w) \\
& =\max \{d(A z, S z), d(B w, T w), d(B w, A z) \\
& \quad(d(A z, T w) \cdot d(B w, S z))^{1 / 2}, \min \left\{\frac{d(A z, S z) \cdot d(B w, T w)}{d(A z, B w)}\right. \\
& \left.\left.\quad \frac{d(A z, T w) \cdot d(B w, S z)}{d(A z, B w)}, \frac{d(A z, T w) \cdot d(B w, S z)}{d(S z, T w)}\right\}\right\} \\
& =\max \left\{1,1, d(z, w), d(z, w), \min \left\{\frac{1}{d(z, w)}, d(z, w), d(z, w)\right\}\right\} \\
& =d(z, w),
\end{aligned}
$$

which implies that $z=w$. Therefore, $A, B, S$ and $T$ have a unique common fixed point in $X$. This completes the proof.

Finally, we give the following theorem for compatible mappings of type $(E)$.

Theorem 2.3. Let $A, B, S$ and $T$ be mappings of a complete multiplicative metric space $(X, d)$ into itself satisfying the conditions $\left(C_{1}\right),\left(C_{2}\right)$ and $\left(C_{3}\right)$.

Assume that the pairs $A, S$ and $B, T$ are compatible of type $(E)$, Then $A, B, S$ and $T$ have a unique common fixed point in $X$.

Proof. From the proof of Theorem 2.2, $\left\{y_{n}\right\}$ is a multiplicative Cauchy sequence in $X$ and hence it converges to some point $z \in X$. Further the subsequence $\left\{S x_{2 n}\right\},\left\{T x_{2 n+1}\right\},\left\{B x_{2 n+1}\right\}$ and $\left\{A x_{2 n}\right\}$ of $\left\{y_{n}\right\}$ converges to $z$.

Now suppose that $A$ and $S$ are compatible of type $(E)$ and one of mappings $A$ and $S$ is continuous. Then by Proposition 1.8 we have $A z=S z$. Since $S X \subset B X$ so there exists a point $u \in X$ such that $S z=B u$.

Now, putting $x=z$ and $y=u$ in $\left(C_{2}\right)$, we have

$$
d(S z, T u) \leq M^{\lambda}(z, u)
$$


where

$$
\begin{aligned}
& M(z, u) \\
& =\max \{d(A z, S z), d(B u, T u), d(B u, A z), \\
& \quad(d(A z, T u) \cdot d(B u, S z))^{1 / 2}, \min \left\{\frac{d(A z, S z) \cdot d(B u, T u)}{d(A z, B u)},\right. \\
& \left.\left.\quad \frac{d(A z, T u) \cdot d(B u, S z)}{d(A z, B u)}, \frac{d(A z, T u) \cdot d(B u, S z)}{d(S z, T u)}\right\}\right\} \\
& =d(S z, T u) .
\end{aligned}
$$

Hence we have

$$
d(S z, T u) \leq d^{\lambda}(S z, T u)
$$

which implies that $S z=T u$ and hence we have $A z=S z=T u=B u$.

Next putting $x=z$ and $y=x_{2 n+1}$ in $\left(C_{2}\right)$, we have

$$
d\left(S z, T x_{2 n+1}\right) \leq M^{\lambda}\left(z, x_{2 n+1}\right),
$$

where

$$
\begin{aligned}
& M\left(z, x_{2 n+1}\right) \\
& =\max \left\{d(A z, S z), d\left(B x_{2 n+1}, T x_{2 n+1}\right), d\left(B x_{2 n+1}, A z\right)\right. \\
& \left(d\left(A z, T x_{2 n+1}\right) \cdot d\left(B x_{2 n+1}, S z\right)\right)^{1 / 2}, \min \left\{\frac{d(A z, S z) \cdot d\left(B x_{2 n+1}, T x_{2 n+1}\right)}{d\left(A z, B x_{2 n+1}\right)},\right. \\
& \left.\left.\quad \frac{d(A z, T y) \cdot d\left(B x_{2 n+1}, S z\right)}{d\left(A z, B x_{2 n+1}\right)}, \frac{d\left(A z, T x_{2 n+1}\right) \cdot d\left(B x_{2 n+1}, S z\right)}{d\left(S z, T x_{2 n+1}\right)}\right\}\right\} .
\end{aligned}
$$

Taking $n \rightarrow \infty$, we get

$$
\begin{aligned}
& \lim _{n \rightarrow \infty} M\left(z, x_{2 n+1}\right) \\
& =\max \left\{d(S z, S z), d(z, z), d(z, S z),(d(S z, z) \cdot d(z, S z))^{1 / 2},\right. \\
& \left.\quad \min \left\{\frac{1}{d(S z, z)}, d(S z, z), d(S z, z)\right\}\right\} \\
& =d(S z, z) .
\end{aligned}
$$

This implies that $S z=z$. Hence, $z$ is a common fixed point of $A$ and $S$. 
Again, suppose that $B$ and $T$ are compatible of type $(E)$ and one of mappings $B$ and $T$ is continuous we get $B u=T u=A z=z$. By Proposition 1.8 we have $B B u=B T u=T B u=T T u$, that is, $B z=T z$.

Next putting $x=x_{2 n}$ and $y=z$ in inequality $\left(C_{2}\right)$, we have

$$
d\left(S x_{2 n}, T z\right) \leq M^{\lambda}\left(x_{2 n}, z\right),
$$

where

$$
\begin{aligned}
& M\left(x_{2 n}, z\right) \\
& =\max \left\{d\left(A x_{2 n}, S x_{2 n}\right), d(B z, T z), d\left(B z, A x_{2 n}\right),\right. \\
& \quad\left(d\left(A x_{2 n}, T z\right) \cdot d\left(B z, S x_{2 n}\right)\right)^{1 / 2}, \min \left\{\frac{d\left(A x_{2 n}, S x_{2 n}\right) \cdot d(B z, T z)}{d\left(A x_{2 n}, B z\right)},\right. \\
& \left.\left.\quad \frac{d\left(A x_{2 n}, T z\right) \cdot d\left(B z, S x_{2 n}\right)}{d\left(A x_{2 n}, B z\right)}, \frac{d\left(A x_{2 n}, T z\right) \cdot d\left(B z, S x_{2 n}\right)}{d\left(S x_{2 n}, T z\right)}\right\}\right\} .
\end{aligned}
$$

Letting $n \rightarrow \infty$, we get

$$
\begin{aligned}
& \lim _{n \rightarrow \infty} M\left(x_{2 n}, z\right) \\
& =\max \left\{1,1, d(T z, z), d(T z, z), \min \left\{\frac{1}{d(z, T z)}, d(z, T z), d(T z, z)\right\}\right\} \\
& =d(T z, z) .
\end{aligned}
$$

This implies that $T z=z$ and hence we have $T z=B z=z$. Hence, $z$ is a common fixed point of $B$ and $T$. Thus, $z$ is a common fixed point of $A, B, S$ and $T$.

Uniqueness follows easily. Therefore, $A, B, S$ and $T$ have a unique common fixed point in $X$. This completes the proof.

\section{References}

[1] A.E. Bashirov, E.M. Kurplnara, A. Ozyapici, Multiplicative calculus and its applicatiopns, J. Math. Anal. Appl., 337 (2008), 36-48, doi: 10.1016/j.jmaa.2007.03.081.

[2] S.M. Kang, P. Kumar, S. Kumar, P. Nagpal, S.K. Garg, Common fixed points for compatible mappings and its variants in multiplicative metric spaces, Int. J. Pure Appl. Math., 102 (2015), 383-406, doi: 10.12732/ijpam.v102i2.14.

[3] S.M. Kang, P. Kumar, S. Kumar, Common fixed points for compatible mappings of types in multiplicative metric spaces, Int. J. Math. Anal., 9 (2015), 1755-1767, doi: 10.12988/ijma.2015.53104. 
[4] P. Kumar, S. Kumar, S.M. Kang, C.Y. Jung, Fixed points for compatible mappings in multiplicative metric spaces, Int. J. Pure Appl. Math., 109 (2016), 619-629, doi: 10.12732/ijpam.v109i3.11.

[5] Y.C. Kwun, P. Kumar, S.M. Kang, S. Kumar, Some common fixed point results for compatible mappings of types in multiplicative metric spaces, Int. J. Pure Appl. Math., 108 (2016), 141-158, doi: 10.12732/ijpam.v108i1.13.

[6] M. Özavsar, A. C. Çevikel, Fixed points of multiplicative contraction mappings on multiplicative metric spaces, ArXiv:1205.5131v1. 
